structural concept for the polymer system because of their tailoring properties. Oligo( $\varepsilon$-caprolactone)diol (OCL), which is responsible for switching segments that determine both the temporary and permanent shape of the polymer, initiated a ring-opening polymerization of cyclic diesters or lactones to make macrodiols first, then two macrodiols were coupled with 2,2(4),4-trimethylhexanediisocyanate to form the multiblock copolymers. The crystallizable oligo( $p$-dioxanone)diol (ODX) was the hard segment in the polymer network. By varying the ratio of OCL and ODX, the cross-link density of the polymer can be adjusted. Thus, the mechanical strength and transition temperature of the polymers can be tailored over a wide range, which offered the materials "excellent" shape-memory properties. A series of tests confirmed that the materials can be programmed into permanent and temporary shapes in seconds with up to $400 \%$ deformation, while the maximum deformation for Nitinol is just $8 \%$; the new materials can be adjusted at a lower transition temperature than metal alloys; and the introduced hydrolyzable ester bonds in the polymers will break under physio- logical conditions, which provides an additional advantage (biodegradability) over Nitinol.

The researchers said that the shapememory capability of the polymer materials enables bulky implants to be placed in the body through small incisions. These materials can also perform complex mechanical deformations automatically. Another possible application, the researchers said, is the design of a smart surgical suture that allows an optimized tightening process of the knot.

Yue Hu

\section{Abrasive Jet Machining of Brittle Ceramics Yields Smoother Surfaces than Conventional Finishing Methods}

The most commonly used technique for surface finishing of ceramic components is grinding by a diamond wheel. However, this method is not ideal for brittle ceramics such as alumina and silicon carbide. A group of researchers at the Fine Ceramics Research Association and the National Institute of Advanced Industrial Science and Technology, Nagoya, have found that the use of abrasive jet machining (AJM) on alumina samples resulted in a much smoother surface and a significant improvement in flexural strength as compared with the same samples processed by conventional methods. AJM is a specialized form of shot blasting, featuring the use of fine, hard, abrasive particles projected at an extremely high velocity.

As described in the May issue of the Journal of the American Ceramics Society, Manabu Wakuda of the Association and co-workers prepared three sets of samples of the same dimensions by machining the alumina ceramic. One set was ground with a diamond wheel. Another set was subsequently finished by lapping with diamond pastes after grinding. The third set was finished by the AJM process, which uses a pressurized nitrogen stream containing silicon carbide particles in the 15-25- $\mu \mathrm{m}$ range.

After the processes, the researchers evaluated material removal behavior, mechanical properties, and residual stresses at the machined surface of these samples. The surface of the ground sample appeared relatively rough, with grains exposed. The surface following AJM displayed a much smoother topography, without revealing individual grains. This indicates that the material removal in grinding was domi-

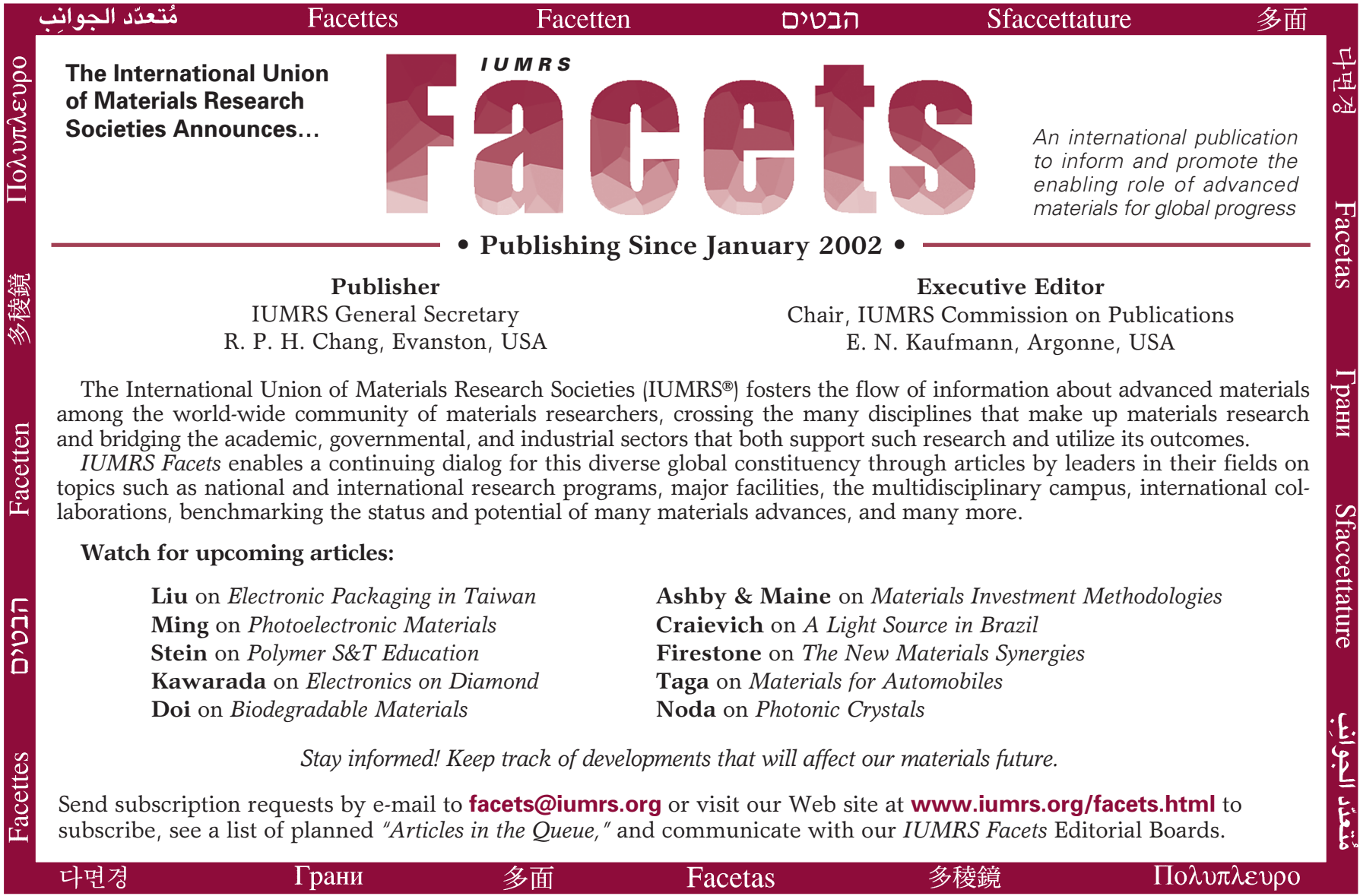

Circle No. 13 on Inside Back Cover 
nated by intergranular fracture, while in AJM it was in a manner resembling ductile behavior. Improved surface finishing by AJM resulted in a $15 \%$ improvement in flexural strength, compared with both ground and ground-plus-lapped samples. The researchers concluded that a higher compressive residual stress observed on the AJM-processed surface, combined with the smoother surface and a reduction in machining flaws, is responsible for the enhancement of the flexural strength.

SHIMING WU

\section{Ceramic-Based Anode for Solid-Oxide Fuel Cell Utilizes Higher-Weight Hydrocarbon Fuels without Coking}

Solid-oxide fuel cells (SOFCs) based upon hydrocarbon fuels are susceptible to loss of performance due to the deposition of carbon, a process known as coking. The standard anodes in many SOFCs contain high levels of Ni, which is known to promote coking. However, recent studies performed on a ceramic-based anode have led to a method that minimizes the amount of nickel required for SOFCs, so that electrochemical reactions are catalyzed while minimizing the amount of carbon deposited. In the June issue of Electrochemical and Solid-State Letters, Zhiqiang Ji of Applied Thin Films Inc. in Evanston, Ill., and Juang Liu, Brian D. Maddison, and Scott A. Barnett of Northwestern University report that by altering the composition of an electronic conductor, $\mathrm{La}_{0.8} \mathrm{Sr}_{0.2} \mathrm{Cr}_{0.8} \mathrm{Mn}_{0.2} \mathrm{O}_{3-\delta}$ (LSCM), by mixing it with an ionic conductor, $\mathrm{Ce}_{0.9} \mathrm{Gd}_{0.1} \mathrm{O}_{1.95}$ (GDC), the threephase boundaries increase and thus enable hydrocarbon oxidation without coking.

Multilayer fuel-cell pellets were manufactured by pressing GDC powder into pellet form and sintering the pellets at $1500^{\circ} \mathrm{C}$ for $6 \mathrm{~h}$. The anode was made by mixing metal oxides of lanthanum, strontium, chromium, and manganese in water prior to ballmilling for $24 \mathrm{~h}$, followed by calcining at $1100^{\circ} \mathrm{C}$ for $2 \mathrm{~h}$. After drying and grinding, the powder was mixed with $50 \mathrm{wt} \%$ GDC, $5 \mathrm{wt} \% \mathrm{NiO}$, water, and polyvinyl alcohol, ground and then painted on one side of the GDC pellet and sintered at $1100^{\circ} \mathrm{C}$ for $3 \mathrm{~h}$. The $\mathrm{NiO}$ had a 16-nm average particle size, while the average particle size of the GDC was $50 \mathrm{~nm}$. An anode with $50 \mathrm{wt} \% \mathrm{Ni}$ and $50 \mathrm{wt} \%$ GDC was also fabricated in a similar fashion for comparison. The cathode consisted of $50 \mathrm{wt} \% \mathrm{La}_{0.6} \mathrm{Sr}_{0.4} \mathrm{Co}_{0.2} \mathrm{Fe}_{0.8} \mathrm{O}_{3-\delta}$ (LSCF) and $50 \mathrm{wt} \%$ GDC synthesized in the method described and sintered at $900^{\circ} \mathrm{C}$ for $3 \mathrm{~h}$. Both the anode and the cathode were $\sim 20 \mu \mathrm{m}$ thick, with an area of $0.3-0.6 \mathrm{~cm}^{2}$.
In tests, both the LSCM-GDC-Ni and $\mathrm{Ni}-\mathrm{GDC}$ anodes performed about the same in hydrogen; however, when tested with propane, the power density was larger for the LSCM-GDC-Ni anode. No obvious carbon deposits were seen on the LSCM-GDC-Ni anode, but the GDC-Ni anode was heavily covered. As expected for SOFCs, power density increased as temperature increased, with a proportional decrease in cell resistance. Final results show that LSCM, although a poor catalyst for hydrocarbon oxidation, acts as the electronic conductor and the mechanical support, while the nickel acts as the oxidation catalyst.

DONALD CARTER

\section{Polymeric Additive Provides Morphological Control of Calcium Oxalate Dihydrate Crystals}

Control of crystal morphology and size are well-known approaches to solving many problems in various areas of materials, including catalysis, medicines, electronics, ceramics, pigments, and cosmetics. Researchers from the College of Chemistry at Peking University in Beijing have discovered a method for morphology-controlled synthesis of calcium oxalate dihydrate (COD). As reported in the June 17 issue of Chemistry of Materials, Dongbai Zhang and co-workers at Peking have described a technique for the preparation of tetragonal rodlike prisms using a double-hydrophilic block copolymer.

The researchers performed the crystallization of calcium oxalate in aqueous solutions of a double-hydrophilic block copolymer of poly(ethylene glycol)-block-poly (methacrylic acid) (PEG-b-PMAA) at different PEG- $b$-PMAA and $\mathrm{Ca}^{2+}$ concentrations, as well as at different $\left[\mathrm{Ca}^{2+}\right] /\left[\mathrm{C}_{2} \mathrm{O}_{4}{ }^{2-}\right]$ ratios. The morphology of COD crystals was characterized by x-ray diffraction and scanning electron microscopy. The researchers observed that the increase of polymer concentration changes the morphology of resulting COD crystals from tetragonal bipyramids dominated by the $\{101\}$ faces to rodlike tetragonal prisms dominated by the $\{100\}$ faces, which is a morphology adopted by some plant COD crystals but not obtained in vitro previously. The COD square prisms obtained in different experiments ranged in size from side lengths of $0.6-2.3 \mu \mathrm{m}$ and side widths of $0.2-1.4 \mu \mathrm{m}$, resulting in the maximal aspect ratio of $\sim 3.5$.

The researchers suggested a latticematching model (based on the similarity of COD unit-cell parameters and distances between two terminal groups in a PMAA chain) to explain the specific morphological influence of PEG- $b$-PMAA on COD crystals. Such a model was extensively used to explain the interaction between crystal faces of inorganic compounds and organic templates/additives. The researchers tested the proposed model on the crystallization of strontium oxalate in the presence of PEG- $b$-PMAA under similar conditions, and found that the suggested model works as expected in this case.

The researchers said that the observed morphological control of COD crystals by the polymeric additive provides insights into the specific function of biomacromolecules in the morphological control of plant COD crystals and could be applied to other synthetic systems of interest.

ANDREI A. ELISEEV

\section{Addition of Fumed Silica Nanoparticles Enhances the Permeability of a Polymer Reverse-Selective Membrane}

Recently developed, reverse-selective membranes offer improved industrial refinement of natural gas, polyolefin, and hydrogen through their counterintuitive property of preferentially permeating large organic molecules over smaller gases. T.C. Merkel and co-workers of the Research Triangle Institute in North Carolina fabricated membranes with increased permeability and selectivity by adding nanometer-scale, nonporous, fumed silica particles to a glassy, lowdensity polyacetylene-such as poly(4methyl-2-pentyne) (PMP) - matrix, as reported in the April 19 issue of Science.

The permeability of a membrane is defined as the product of the penetrant solubility and diffusivity, while the selectivity of gas A over gas B is represented by the ratio of their respective permeabilities. Higher-molecular-weight hydrocarbon gases exhibit greater solubility than lighter gases because they are more readily condensed into liquids for transport through the membrane. However, the larger hydrocarbon chains generally diffuse much more slowly than the smaller, lighter materials. The diffusivity effect usually dominates, and conventional membranes permeate smaller molecules, such as hydrogen, more effectively than larger ones, such as methane. However, this size-sieving diffusion contribution is minimized for membranes with high free volumes because they contain diffusive pathways that transport large molecules nearly as efficiently as smaller ones. Such membranes exhibit the reverse-selective behavior that purifies natural gas by permitting the efficient removal of higher hydrocarbons from methane.

Previous attempts to improve membrane selectivity focused on the addition of 